$(61.3 \pm 30.9 \%)$, KCO $1.05 \pm 0.40 \mathrm{mmol} / \mathrm{min} / \mathrm{kPa} / \mathrm{L}(66.4 \pm$ $22.2 \%)$ and RV $3.58 \pm 1.36 \mathrm{~L}(171.8 \pm 50.4 \%)$. The mean FIB4 was $1.76 \pm 1.36$. Eight $(26.7 \%)$ patients had liver disease on USS. FIB4 cut-off values of 1.45 and 3.25 were utilised, as they are widely validated. ${ }^{3}$ Of patients $>3.25,100 \%$ had abnormal scans (PPV 100\%), and of patients $<1.45,15.8 \%$ had abnormal scans (NPV 82\%). FIB4 enabled correct identification of patients with abnormal USS with an area under a ROC curve of 0.642. We demonstrate a relationship between liver involvement in A1ATD and BMI. Those with higher FIB4 had higher BMI $(r=0.453, p=0.008)$. We found no relationship between FIB4 and severity of lung involvement.

Conclusions The FIB4 score, calculated from routine laboratory variables and age, may be useful to rule in and out significant liver involvement in A1ATD with reasonable sensitivity and specificity. Further work is required for validation against biopsy, the gold-standard assessment. We confirm a previously noted lack of association between liver disease and emphysema severity, ${ }^{4}$ and highlight the association between higher BMI and higher fibrosis risk in A1ATD.

\section{P60 CANNABIS LUNG CAUSING DEBILITATING EMPHYSEMA: ARE WE ON THE VERGE OF AN EPIDEMIC?}

Narendra Babu Chinnappa, Kasia Zalewska, Damian Mckeon. Ysbyty Gwynedd, Bangor, UK

\subsection{6/thoraxjnl-2014-206260.201}

Introduction and objectives Cannabis (or marijuana) is the world's most widely-used illicit drug, according to UN drug report 2012 prevalence of cannabis use between 15-64 years of age is around $1.7 \%$ in Europe and $2.6 \%$ in USA. ${ }^{1}$ It is particularly prevalent amongst adolescents and young adults. As societies reconsider the legal status of cannabis, policy makers and clinicians require sound knowledge of the acute and chronic effects of cannabis. There has been surprisingly little research into its effects on respiratory health. In a rural region of North Wales we have noticed an increasing number of young patients presenting with precocious bullous emphysema associated with very high tobacco and cannabis usage.

Methods A series of 8 patients presenting through the Emergency Department with an exacerbation of COPD were noted to have precocious COPD associated with high cannabis use. The age was between 35-48, all had both physiological and radiological signs of advanced emphysema. All had at least 10-20 years of cannabis usage smoking more than 5 'joints' per day. Of these, 4 patients were significantly impaired to require long term oxygen therapy, and one is actively listed for a single lung transplant. All had normal levels of alpha 1 antitrypsin and chymotrypsin.

Results We found young patients with debilitating COPD secondary to cannabis use i.e. as less as 10 years of use. ${ }^{2}$ We postulate that cannabis smoking leads to severe COPD in young patients independent of genetic susceptibility, which is on the verge of increase.

Conclusions The addition of cannabis to the tobacco, and high usage at a young age is leading to increase in the incidence of COPD in general and bullous emphysema as a phenotype in particular. We are concerned that the dangers of cannabis inhalation and these risks are not being appreciated by the wider health community. More research is needed to know the mechanisms of the inflammatory response secondary to cannabis smoking.

\section{REFERENCES}

1 UN drug report 2012, United Nations Office on Drugs and Crime

2 Tashkin DP. Effects of marijuana smoking on the lung. Annals of the American Thoracic Society. 2013;10(3):239-47 \begin{tabular}{l}
\hline P61 THE PREVALENCE OF HYPERCAPNIA IN PATIENTS WITH \\
ALPHA-1-ANTI-TRYPSIN DEFICIENCY (AATD)
\end{tabular}

C Dave, A Turner, T Spruell. Birmingham HeartlandsHospital, Birmingham, UK

10.1136/thoraxjnl-2014-206260.202

Introduction Hypercapnia in the acute phase of COPD exacerbation is common, with $\mathrm{CO}_{2}>6 \mathrm{kPA}$ in $44 \%$ of patients at some point during their admission. Little data exists on the prevalence of hypercapnia in stable COPD patients, and even less in those with AATD. As emphysema is more predominant in the lower lobes of AATD patients, this is likely to contribute to hyperinflation and hence potentially increase $\mathrm{CO}_{2}$ levels.

Methods The Birmingham AATD database (ADAPT) is a registry of with over 1000 patients with AATD. The registry has basic demographics, detailed spirometric parameters as well as baseline blood gases. Hypercapnia is defined as $\mathrm{CO}_{2}$ greater than $5.5 \mathrm{kPa}$.

Results The blood gas results of 766 (PiZZ genotypes) individual patients were available for analysis. 93 patients (12.14\%) had a type 1 respiratory failure, defined as a $\mathrm{PO}_{2}<8 \mathrm{kPa}, 69$ had hypercapnia (9.01\%) and $16(2.09 \%)$ patients fulfilled both criteria. There is a statistically significant difference seen in the hypercapnic vs nonhypercapnic population with regards to $\mathrm{FEV}_{1}$ (1.07 vs $1.46, \mathrm{p}=$ $0.01)$, FVC (3.45(CI 3.1-3.81) vs 3.82, $\mathrm{p}=0.02)$ and BMI (27.1 vs $24.9, \mathrm{p}=0.02)$. There is no difference in the amount of upper zone emphysema (29.54 vs 30.50 (CI 29.12-33.01)) or lower zone emphysema (40.66 vs 49.13 (CI 42.64-47.32)). Chi-squared analysis of lower zone predominance (lower zone - upper zone) showed no statistical difference either $(p=0.76)$. Factors clinically significant in univariate analysis were taken forward to logistic regression analysis where BMI was the only clinically significant $(\mathrm{p}=0.008)$ predictor. Conclusion Hypercapnia is relatively common amongst AATD patients, but Type 2 respiratory failure is uncommon. There is an increased risk of hypercapnia with worse $\mathrm{FEV}_{1}, \mathrm{FVC}$ and higher BMI. The presence or location of emphysema did not seem to influence the $\mathrm{CO}_{2}$ levels.

\section{P62 CORRELATION OF QUANTITATIVE CHEST CT MEASURES WITH LUNG FUNCTION AND FUNCTIONAL PARAMETERS IN A COHORT OF MODERATE TO VERY SEVERE COPD PATIENTS}

${ }^{1} \mathrm{~K}$ Ostridge, ${ }^{1} \mathrm{~N}$ Williams, ${ }^{1} \mathrm{~V}$ Kim, ${ }^{1} \mathrm{~A}$ Barton, ${ }^{2} \mathrm{MM}$ Wojtas, ${ }^{3} \mathrm{~S}$ Harden, ${ }^{4} \mathrm{E}$ Aris, ${ }^{4} \mathrm{M}$ Peeters, ${ }^{4} \mathrm{JM}$ Devaster, ${ }^{5} \mathrm{~S}$ Bourne, ${ }^{6} \mathrm{~T}$ Wilkinson. ${ }^{1}$ Southampton NIHR Respiratory Biomedical Research Unit, UniversityHospital Southampton Foundation NHS Trust, Southampton, UK; ${ }^{2}$ Southampton NIHR Biomedical Research Centre, Southampton, UK; ${ }^{3}$ Department of Radiology, University Hospitals Southampton NHS Foundation Trust, Southampton, UK ; ${ }^{4}$ GlaxoSmithKline Vaccines, Rixensart, Belgium; ${ }^{5}$ Department of Respiratory Medicine, University Hospitals Southampton NHS Foundation Trust, Southampton, UK; ${ }^{6}$ Faculty of Medicine, University of Southampton, Southampton, UK

\subsection{6/thoraxjnl-2014-206260.203}

Introduction COPD is a heterogeneous condition consisting of a number of different clinico-pathological subgroups (phenotypes), leading to particular challenges in managing the condition. Recognising these phenotypes may assist in directing the choice of treatment options. CT is being investigated as a tool for identifying key morphological features seen in COPD. Computer 